\title{
Erector Spinae Plane Block for Lumbar Spinal Surgery: A Systematic Review
}

This article was published in the following Dove Press journal:

Journal of Pain Research

\author{
Yong Qiu \\ Teng-Jiao Zhang (D) \\ Zhen Hua (D) \\ Anesthesiology Department, Beijing \\ Hospital, National Center of \\ Gerontology, Institute of Geriatric \\ Medicine, Chinese Academy of Medical \\ Sciences, Beijing 100730, People's \\ Republic of China
}

Background: Erector spinae plane block (ESPB) as a new trunk fascia block technique was proposed in 2016. ESPB has aroused the interest of many nerve block experts. However, there are few clinical studies on ESPB for lumbar surgery, and its effectiveness and safety are controversial. The goal of this review is to summarize the use of ESPB for lumbar spine surgery in order to better understand this technique.

Methods: PubMed, EMBASE, Cochrane library and ClinicalTrial.gov databases were searched up to July 30, 2019. According to the inclusion and exclusion criteria established in advance, "lumbar spine surgery" and "ESPB" related MesH terms and free-text words were used. Data on pain scores, analgesic consumptions and adverse effects were reported. All processes follow PRISMA statement guidelines.

Results: A total of 171 participants from 11 publications were identified, including two randomized controlled trials (RCTs), one retrospective cohort study, four case reports and four cases series. Block operation planes from T8 to L4. The main anesthetics used in the block are bupivacaine, ropivacaine and lidocaine. There was evidence for reducing postoperative pain scores and analgesic consumptions.

Conclusion: The effectiveness and safety of ESPB for lumbar spine surgery are still controversial. The current evidence is insufficient to support the widespread use of ESPB for lumbar spine surgery. High-quality RCTs are urgently needed.

Keywords: ESPB, lumbar spine surgery, postoperative analgesia

\section{Introduction}

Postoperative pain is often severe in patients undergoing lumbar surgery. Due to postoperative pain, patients are unwilling to get out of bed at an early stage, which affects their recovery. ${ }^{1,2}$ Patient-controlled analgesia or epidural injection analgesia is usually used in the clinic. However, patient-controlled analgesia is prone to opioid-related side effects. Epidural injection is associated with infections, hematomas and other adverse events. ${ }^{3,4}$ Furthermore, the analgesic effect of conventional postoperative analgesia is limited. If the postoperative pain of the lumbar spine could not be effectively relieved, it may develop into chronic pain, affecting the quality of life of the patients. ${ }^{5}$

Erector spinae plane block (ESPB) as a new trunk fascia block technique was proposed in $2016 .^{6}$ ESPB has aroused the interest of many nerve block experts. The benefits of ESPB are not yet demonstrated. The specific mechanism is still controversial. Some believethat ESPB can block the posterior root of the spinal nerve and produce part of the paraspinal block effect with the diffusion of the drug solution. ${ }^{7,8}$ Many scholars have applied ESPB to postoperative analgesia in chest and abdomen. Furthermore, they found that ESPB may reduce
Anesthesiology Department, Beijing

Hospital, National Center of

Gerontology, No. I Dahua Road, Dong

Dan, Beijing 100730, People's Republic of

China

Email huazhenbj528@I63.com 
perioperative muscle relaxation and analgesic drug use. Finneran and co-workers reported that ESPB can provide analgesia for breast surgery. ${ }^{9}$ A study reported that ESPB was effective for abdominal analgesia in weight loss surgery. ${ }^{10}$ Similarly, a report showed that ESPB relieved postoperative pain in patients with lumbosacral spine surgery, reducing the use of analgesic drugs. ${ }^{11}$ Some believed that the analgesic effect of ESPB may be better than that of epidural injection. ${ }^{12}$ Reducing the use of analgesic drugs in the perioperative period is beneficial to accelerate the recovery of patients and reduce the cost of hospitalization.

However, few clinical studies have focused on ESPB in lumbar surgery. What is more, there are differences in the mechanism and effect of block in different parts of the erector spinal muscle. ${ }^{13}$ Some scholars question the practicability of ESPB in lumbar surgery. Tseng and Xu believe that postoperative analgesia in patients with lumbar spine surgery using a thoracolumbar interfascial plane (TILP) block may be better than ESPB. ${ }^{14}$ Therefore, it is necessary to systematically summarize the use of ESPB in lumbar spine surgery so as to better understand and promote this technique and benefit patients undergoing lumbar surgery.

\section{Methods}

\section{Literature Search}

PubMed, EMBASE, Cochrane library,= and ClinicalTrial. gov databases were searched. MeSH terms and free-text words were used, including "lumbar spine surgery", "decompression", "lumbar spinal stenosis", "spondylolisthesis", "ESP block", "erector spinae plane block". The search time was from inception to July 30, 2019. References to relevant articles or reviews were screened to prevent missed inspection. Our retrieval method was developed together with experienced literature retrieval teachers. All the retrieval results were read independently by the two researchers (QY, ZTJ). According to the established inclusion and exclusion criteria, the title, abstract and full text were strictly evaluated, and the basic information included in the article was extracted. Disagreements were resolved by discussing or consulting with another author. All processes followed PRISMA statement guidelines.

\section{Inclusion and Exclusion Criteria Inclusion Criteria}

(1) Study: Case report, case series, retrospective cohort study, and randomized controlled trials (RCTs); (2) participants: lumbar spine surgery patients; (3) interventions: erector spinae plane block.

\section{Exclusion Criteria}

Review.

\section{Data Collection}

Type of publication, year of publication, journal name, authorship country of origin, type of block (single shot, continuous, intermittent bolus), anatomic location, patient age, multimodal analgesia use, opioid consumption, sensory and motor changes, reported single injection techniques, continuous catheters, additives, opioid-related and block-related side effects and adverse events, visual analogue scales (VAS) or numeric rating scales (NRS), patient satisfaction were collected.

\section{Quality Evaluation}

Risk of bias for RCTs were assessed by two researchers using a modified Cochrane risk of bias assessment tool. RCTs were evaluated in terms of random sequence generation; allocation concealment; blinding of patients, clinicians, data collectors, outcome assessors, and data analysts; incomplete outcome data; and selective outcome reporting; other biases. Each potential source of bias was graded as "low risk", "unclear risk" or "high risk". Bias risk map and bias risk summary diagram were generated by RevMan 5.3 (The Cochrane Collaboration, 2014).

\section{Results}

\section{Search results}

A total of 171 participants from 11 publications were identified, including two RCTs, one retrospective cohort study, four case reports and four caseseries. ${ }^{11,15-24}$ The main reasons for the exclusion of articles were nonlumbar surgery or unrelated to the purpose of the study (Figure 1).

\section{Included Literature}

Outcome indicators included morphine consumption, pain scores, adverse reactions and patient satisfaction after lumbar spine surgery. The follow-up period was from 10 hours to 72 hours. Only one article reported that postoperative pain in the lumbar spine was relieved by ESPB and catheterization (placement of a continuous peripheral nerve catheter), and the other 10 articles were treated with bilateral single injection before operation. Block operation plane from $\mathrm{T} 8$ to L4. The main anesthetics used in the block are bupivacaine, 


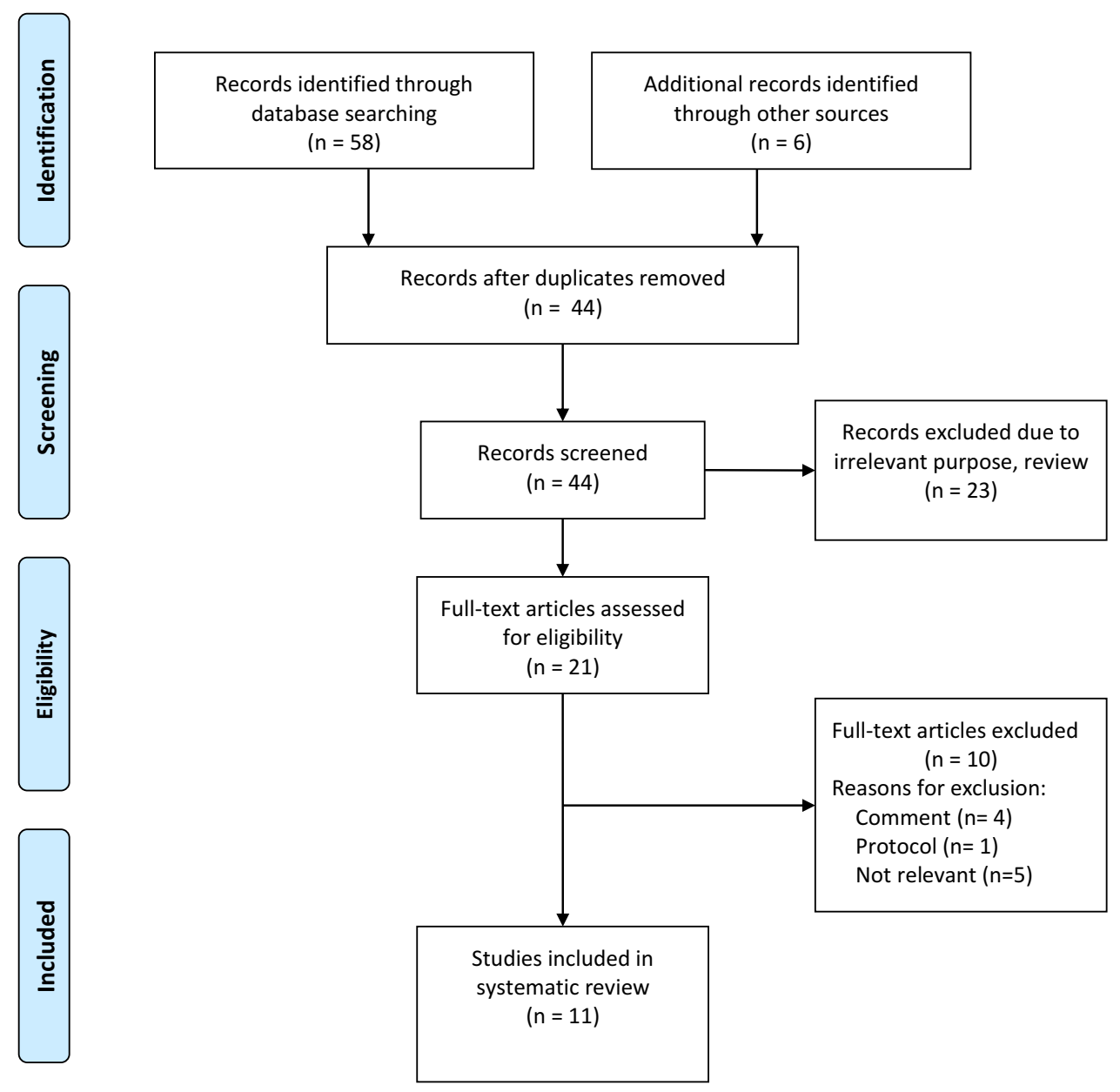

Figure I Flow chart showing the identification and selection of the articles for the systematic review.

ropivacaine and lidocaine. Most of the studies lack a comparator; the effectiveness and safety of ESPB for lumbar spine surgery are still controversial (Table 1).

At the same time, a total of two RCTs were included, but the heterogeneity of outcome indicators could not be analyzed by meta-analysis. Both RCTs found that ESPB significantly reduced lumbar postoperative pain scores and analgesic drug consumption, and no operation-related adverse events occurred. However, the random blind method of the two studies did not do well. Furthermore, small RCTs may not be able to detect adverse effects of therapeutic procedures (Figure 2A and B).

\section{Discussion}

There is an increase in the number of patients with lumbar diseases, and a large number of them need lumbar surgery. ${ }^{25}$ As there is obvious pain after lumbar surgery, postoperative analgesia is often needed. However, patientcontrolled intravenous analgesia and epidural analgesia, which are commonly used in the clinic, have their own shortcomings. ${ }^{4,25}$ Side effects such as nausea and vomiting caused by postoperative opioid use result in poor postoperative experience, reduce patient satisfaction, and are not conducive to rapid recovery. ${ }^{26}$ In fact, neuraxial techniques may be complicated with headache, backache, unintended dural punctures, and puncture site hemorrhage. Neuraxial ultrasound may help improve the safety. Epidural hematoma, epidural abscess and intracord injections are rare but serious complications that need more attention. ${ }^{27}$ In addition, neuraxial techniques have disadvantages such as hypotension, urinary retention and being limited to the patient who has spine fracture or spine surgery. Paraneuraxial nerve blocks such as Erector spinae plane (ESP) block may have an advantage in success rate and analgesic efficacy. ${ }^{28}$ In recent years, many researchers have used ESPB for postoperative analgesia and found that local anesthetic spread well, volume-dependent, and extended into the neural foramina and epidural space 


\begin{tabular}{|c|c|c|c|c|c|c|}
\hline 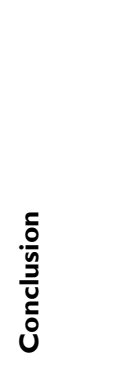 & 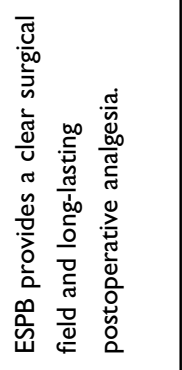 & 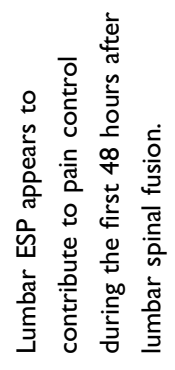 & 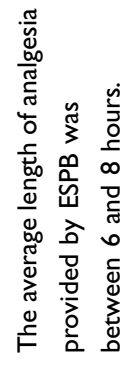 & 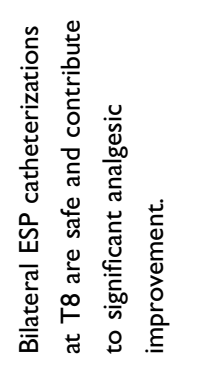 & 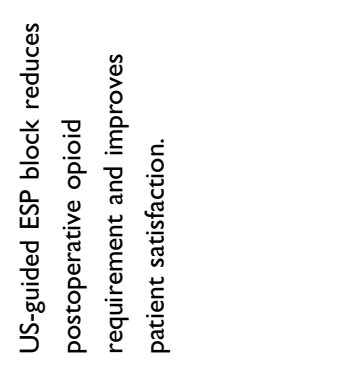 & 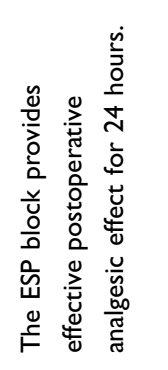 \\
\hline 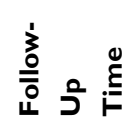 & 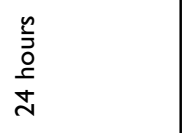 & 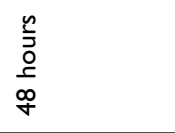 & $\begin{array}{l}\text { ñ } \\
\text { o } \\
\text { 음 }\end{array}$ & 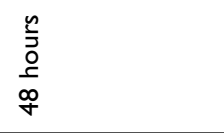 & 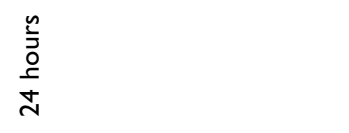 & 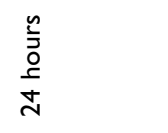 \\
\hline 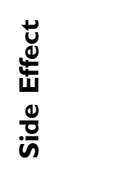 & $\begin{array}{l}0 \\
\stackrel{0}{0} \\
z\end{array}$ & $\begin{array}{l}0 \\
\stackrel{0}{0} \\
z\end{array}$ & $\stackrel{0}{\stackrel{0}{0}}$ & $\begin{array}{l}\stackrel{0}{\tilde{c}} \\
\stackrel{0}{Z}\end{array}$ & 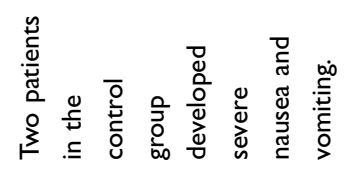 & $\begin{array}{l}0 \\
\stackrel{0}{0} \\
z\end{array}$ \\
\hline 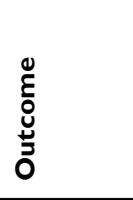 & 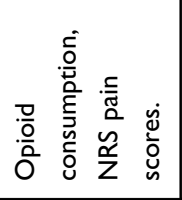 & 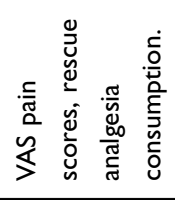 & 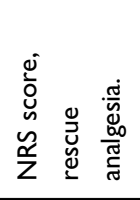 & 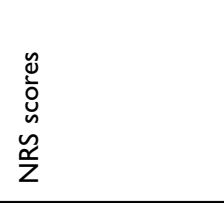 & 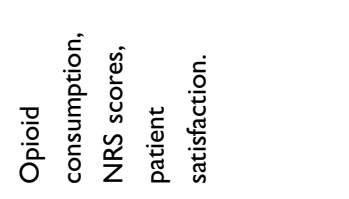 & 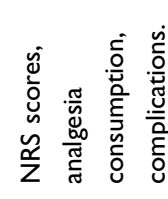 \\
\hline $\begin{array}{l}\infty \\
\frac{0}{u} \\
\text { w }\end{array}$ & 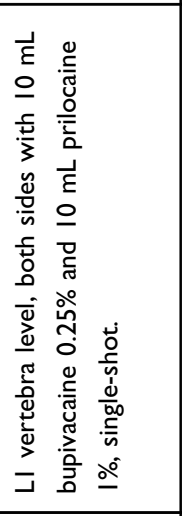 & 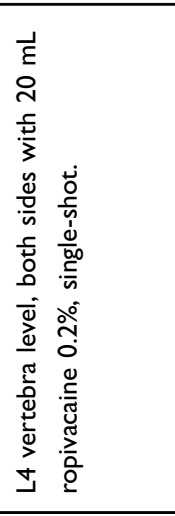 & 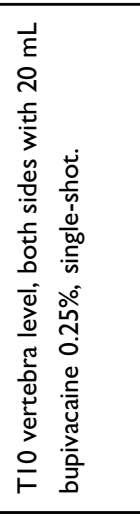 & 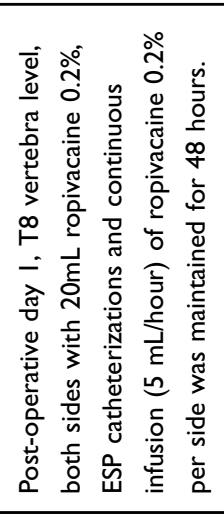 & 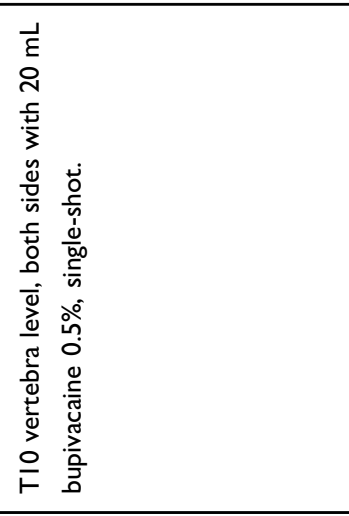 & 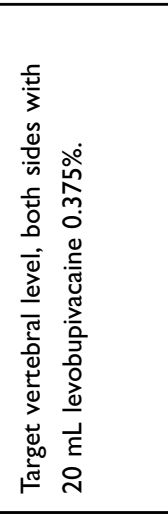 \\
\hline 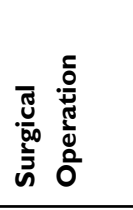 & 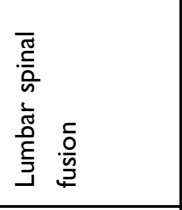 & 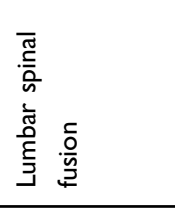 & 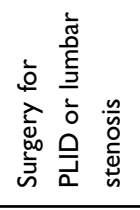 & 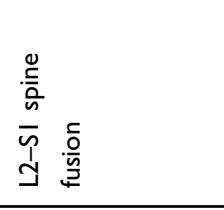 & 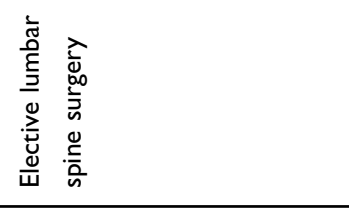 & 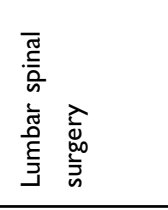 \\
\hline 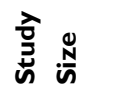 & - & $\infty$ & $\wedge$ & - & 우 & $\bar{\sigma}$ \\
\hline 窟 & 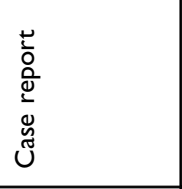 & 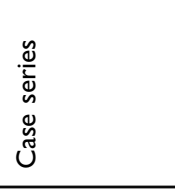 & 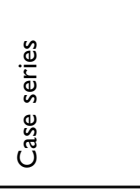 & 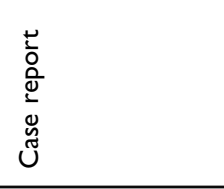 & 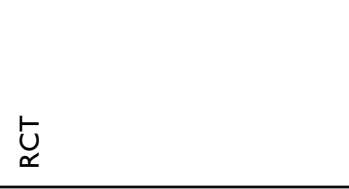 & 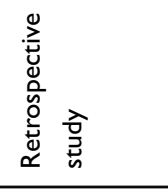 \\
\hline نे & 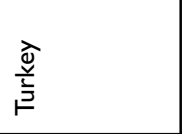 & 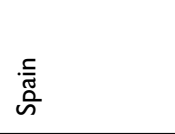 & 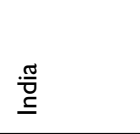 & 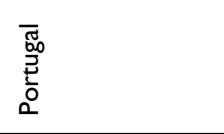 & $\stackrel{\text { 五 }}{\underline{\underline{\underline{T}}}}$ & 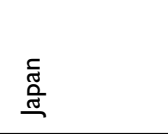 \\
\hline 京 & 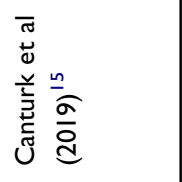 & 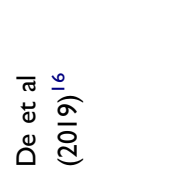 & 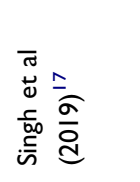 & 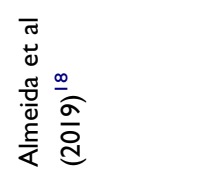 & 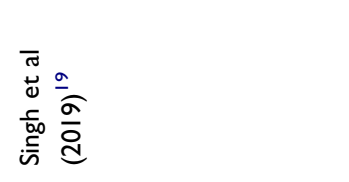 & 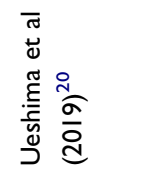 \\
\hline
\end{tabular}




\begin{tabular}{|c|c|c|c|c|}
\hline 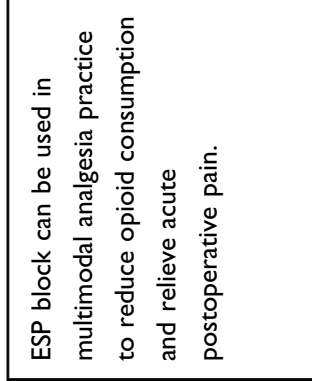 & 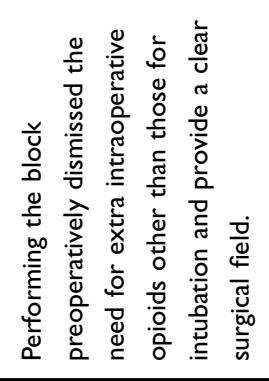 & 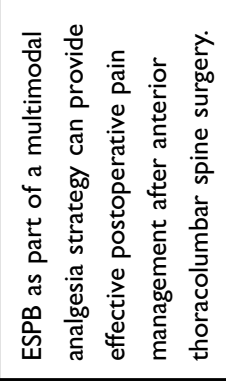 & 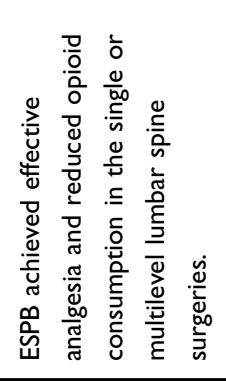 & 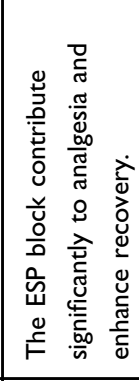 \\
\hline 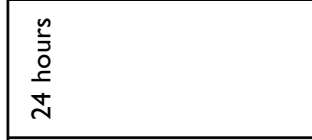 & 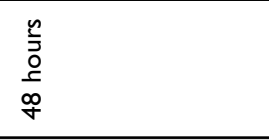 & 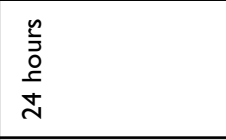 & 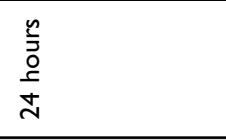 & 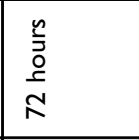 \\
\hline $\begin{array}{l}\frac{0}{0} \\
\text { zo }\end{array}$ & 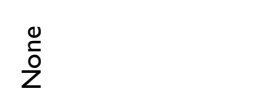 & 总 & 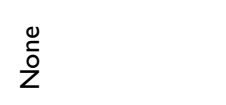 & \begin{tabular}{|l}
0 \\
$\tilde{0}$ \\
$z$
\end{tabular} \\
\hline 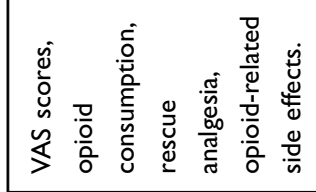 & 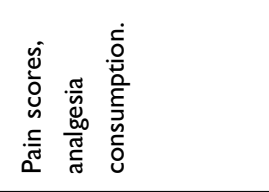 & 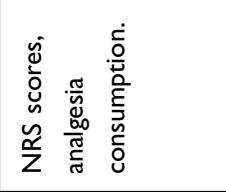 & 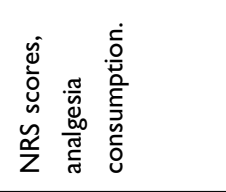 & 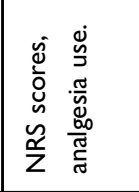 \\
\hline 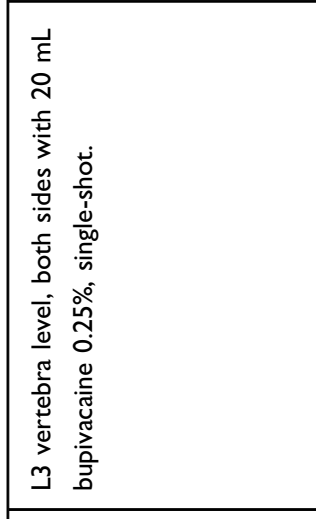 & 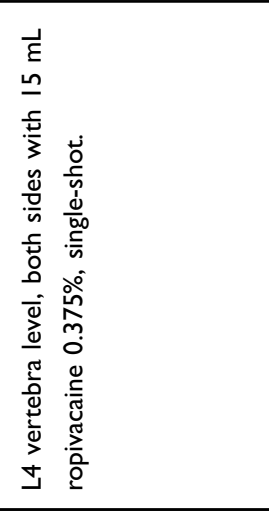 & 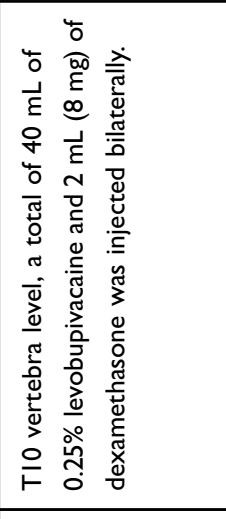 & 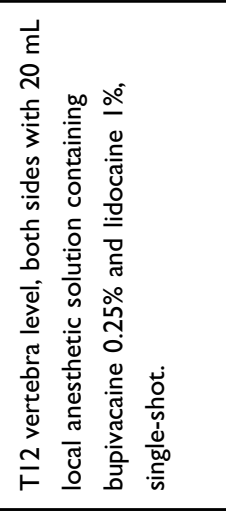 & 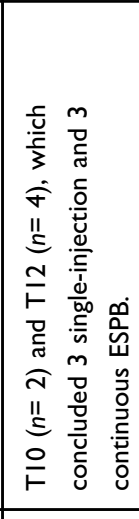 \\
\hline 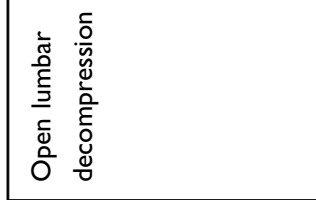 & 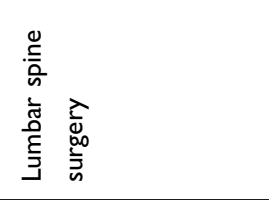 & 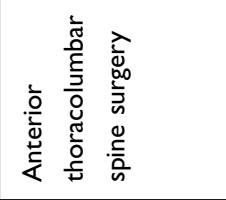 & 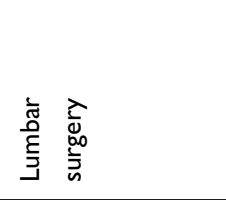 & 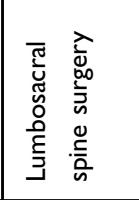 \\
\hline 8 & - & - & in & 0 \\
\hline$\underset{\propto}{\mathfrak{y}}$ & 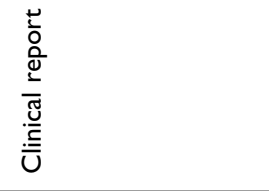 & 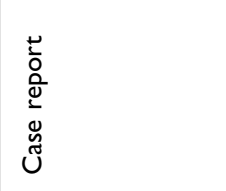 & 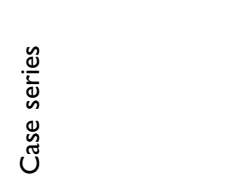 & 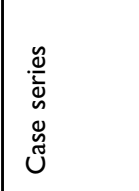 \\
\hline 姟 & 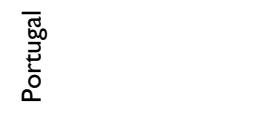 & 颃 & 譶 & 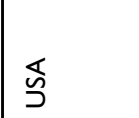 \\
\hline 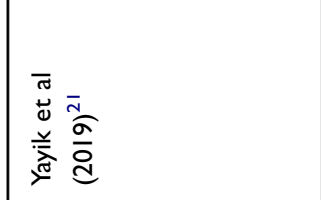 & 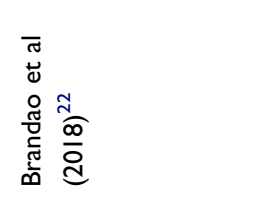 & 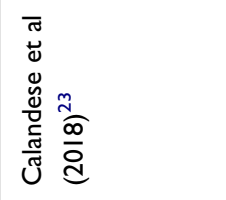 & 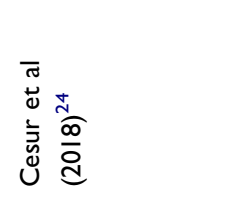 & 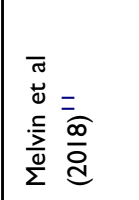 \\
\hline
\end{tabular}




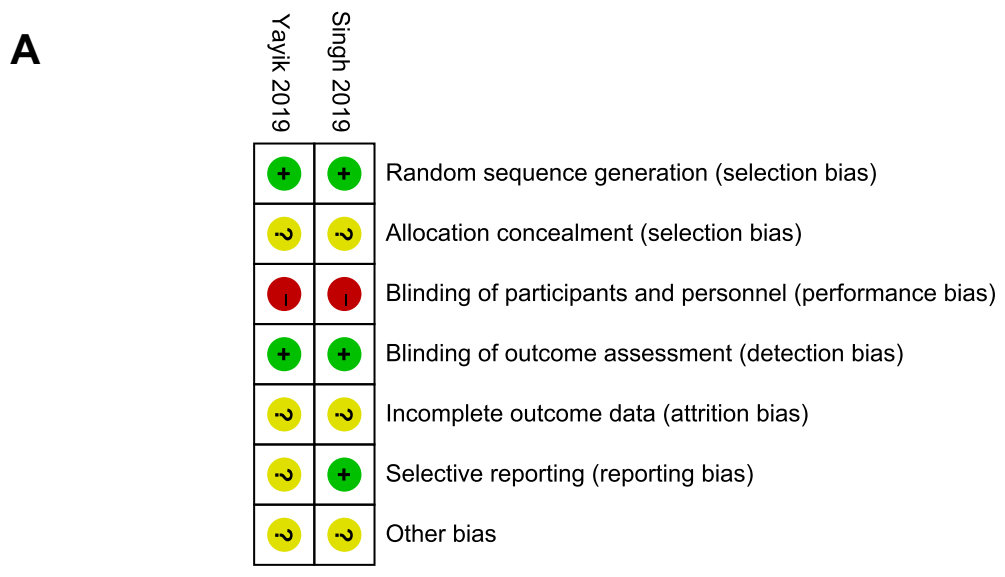

B

Random sequence generation (selection bias)

Allocation concealment (selection bias)

Blinding of participants and personnel (performance bias)

Blinding of outcome assessment (detection bias)

Incomplete outcome data (attrition bias)

Selective reporting (reporting bias)

Other bias

Low risk of bias

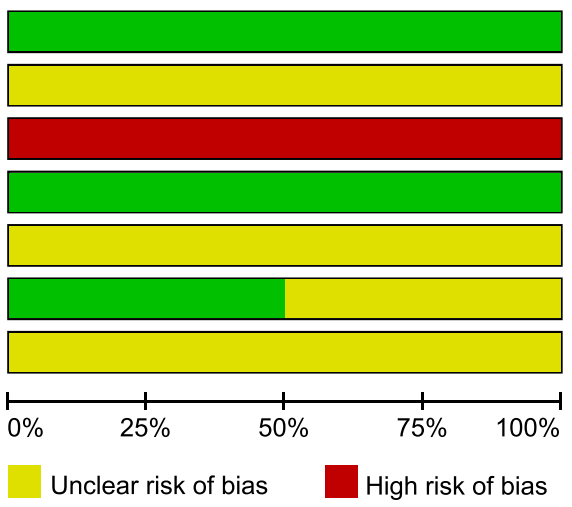

Figure 2 (A) Risk of bias summary: green circles with "+" sign indicate low risk, yellow circles with "?" sign indicate unclear risk, red circles with "-" sign indicate high risk. (B) Risk of bias graph: review authors' judgments about each risk of bias item presented as percentages across all included studies.

normally. At the same time, local anesthetic may show significantly more epidural spread when the lamina and ligaments are compromised, which need more attention. ${ }^{10,29}$ The local anesthetics injected during ESPB spread widely and could produce the effect of a paraspinal block; therefore, the block range was wide and could last until a period of time after operation. ${ }^{14,30,31}$ However, there are few studies on ESPB for postoperative analgesia of lumbar spinae surgery patients. ${ }^{32}$ Therefore, it is necessary to summarize the relevant clinical studies. Eleven studies of ESPB for lumbar surgery were included, and data from 171 patients were extracted and analyzed. Interestingly, two RCTs showed that ESPB may reduce postoperative opioid consumption and pain scores in patients undergoing lumbar surgery. One study showed that ESPB may improve patient satisfaction. However, most of the studies lack a comparator. The effectiveness and safety of ESPB for lumbar spine surgery are still controversial. High-quality RCTs are urgently needed.

It has been reported that pain is obvious at 4 hours after lumbar surgery and relieved after 72 hours. What is more, regional anesthesia can help patients reduce pain and other discomfort. ${ }^{18,33}$ Interestingly, Singh found that when $0.25 \% 20 \mathrm{~mL}$ bupivacaine was injected on both sides of the T10 plane, the 6th hour NRS score was low; however, the 8th hour NRS score was high after lumbar surgery, which suggested that the ESPB could last for 6- $8 \mathrm{~h}$ after operation. ${ }^{34}$ The duration of ESPB block was related to the type and dose of local anesthetics. In addition, local will be washout during the surgery, if ESPB was performed before incision. ${ }^{13}$ However, safety and dosing were not evaluated in any of the included studies. Duration was not assessed as a primary endpoint in the included research. The safe doses of different local anesthetics were different. For example, the unilateral injection volume of ropivacaine was 20 to $40 \mathrm{~mL}$, the concentration was $0.25 \%$ to $0.5 \%$, and the total safe dose was $150 \mathrm{mg}$. It is recommended that each side of bilateral block should be given $0.375 \%$ ropivacaine $20 \mathrm{~mL}$ in adults. ${ }^{15,35}$ Therefore, the dose or concentration can be increased appropriately to prolong the analgesia time and help the patients to get through the most painful stage after operation. At the 
same time, adding other meds to prolong the duration could be helpful. Related high-quality research is necessary and urgently needed.

The puncture plane was from $\mathrm{T} 8$ to $\mathrm{L} 4$, and the followup time was from 10 to $72 \mathrm{~h}$. However, in different studies, different anesthetic techniques were used. Some researchers pay attention to the use of rescue painkillers, while some studies focus on the total amount of postoperative analgesia drugs. ${ }^{14,36}$ Furthermore, different basic analgesia regimens may cause differences in pain scores. As a result, there is great heterogeneity among different studies, and the results cannot be quantitatively synthesized and analyzed. At the same time, it is suggested that we should pay more attention to the primary outcome indicators in the design of clinical trials in the future. The recently reported protocol of ESPB for postoperative analgesia of lumbar surgery is worthy of reference. ${ }^{14}$ At the same time, it is important to note that little attention has been paid to the effects of ESPB on the use of intraoperative analgesic drugs and muscle relaxants. Only one case report mentioned that ESPB could effectively reduce the use of analgesia and muscle relaxant drugs during the perioperative period of spondylolisthesis correction surgery, and appropriate hypotension was beneficial to surgical visual field exposure and operation. ${ }^{37}$ Reducing the use of perioperative anesthetics cannot only reduce the cost of hospitalization, but also reduce the possible side effects of extensive use of anesthetics. At present, some scholars believe that perioperative use of opioids can affect the immune function of patients, and may be associated with the poor prognosis of tumor patients. At the same time, reducing the use of perioperative opioids may reduce the risk of tumor recurrence. ${ }^{38,39}$ From this point of view, it seems that the effect of ESPB on intraoperative opioid dosage is also worthy of attention. On the other hand, the included studies did not seem to pay particular attention to the effect of ESPB on early out-of-bed activity and postoperative hospital stay in patients with lumbar spine surgery. Early out-of-bed activity and early discharge from hospital comply with ERAS, which is also the reason for the promotion of ESPB in patients undergoing lumbar surgery. At the same time, no adverse events related to ESPB were found in the 11 studies. We believe that the clinical research quality of ESPB for lumbar surgery should be improved, and the mechanism of ESPB for postoperative analgesia and perioperative protection of lumbar spine should be further explored.

\section{Limitation}

Although we have strictly formulated the scheme of literature retrieval and data extraction, there are few research reports that can be included. Only two RCT articles were included, and the outcome could not be analyzed. The sample size of the two articles is small, and the random, blind method and research quality need to be improved. Furthermore, most of the studies lack a comparator.

\section{Conclusions}

The effectiveness and safety of ESPB for lumbar spine surgery are still controversial. The current evidence is insufficient to support the widespread use of ESPB for lumbar spine surgery. High-quality RCTs are urgently needed.

\section{Abbreviations}

ESPB, erector spinae plane block; TILP, thoracolumbar interfascial plane; RCTs, randomized controlled trials.

\section{Data Sharing Statement}

The data used and/or analyzed during the current study are available from the corresponding author on reasonable request.

\section{Acknowledgments}

We are thankful to Ling-bing Meng and Meng-lei Hao for their assistance and suggestions during the submitting process.

\section{Author Contributions}

All authors made substantial contributions to conception and design, acquisition of data, or analysis and interpretation of data; took part in drafting the article or revising it critically for important intellectual content; gave final approval of the version to be published; and agree to be accountable for all aspects of the work.

\section{Funding}

Not applicable.

\section{Disclosure}

The authors declare that they have no competing interests.

\section{References}

1. Saleh A, Thirukumaran C, Mesfin A, Molinari RW. Complications and readmission after lumbar spine surgery in elderly patients: an analysis of 2320 patients. Spine J. 2017;17(8):1106-1112. doi:10.1016/j.spinee.2017.03.019 
2. Lin I, Wiles L, Waller R, et al. What does best practice care for musculoskeletal pain look like? Eleven consistent recommendations from high-quality clinical practice guidelines: systematic review. $\mathrm{Br}$ J Sports Med. 2020;54(2):79-86.

3. Naik BI, Nemergut EC, Kazemi A, et al. The effect of dexmedetomidine on postoperative opioid consumption and pain after major spine surgery. Anesth Analg. 2016;122(5):1646-1653. doi:10.1213/ ANE.0000000000001226

4. Gessler F, Mutlak H, Tizi K, et al. Postoperative patient-controlled epidural analgesia in patients with spondylodiscitis and posterior spinal fusion surgery. J Neurosurg Spine. 2016;24(6):965-970. doi:10.3171/2015.8.SPINE15415

5. Hamilton DL, Manickam B. Erector spinae plane block for pain relief in rib fractures. Br J Anaesth. 2017;118(3):474-475. doi:10.1093/bja/ aex013

6. Forero M, Adhikary SD, Lopez H, Tsui C, Chin KJ. The erector spinae plane block: a novel analgesic technique in thoracic neuropathic pain. Reg Anesth Pain Med. 2016;41(5):621-627. doi:10.1097/ AAP.0000000000000451

7. Elsharkawy H, Pawa A, Mariano ER. Interfascial plane blocks: back to basics. Reg Anesth Pain Med. 2018;43(4):341-346. doi:10.1097/ AAP.0000000000000750

8. Hamilton DL, Manickam BP. Is the erector spinae plane (ESP) block a sheath block. Anaesthesia. 2017;72(7):915-916. doi:10.1111/anae. 13912

9. Finneran JJ 4th, Gabriel RA, Khatibi B. Erector spinae plane blocks provide analgesia for breast and axillary surgery: a series of 3 cases. Reg Anesth Pain Med. 2018;43(1):101-102. doi:10.1097/AAP.000 0000000000695

10. Chin KJ, Malhas L, Perlas A. The erector spinae plane block provides visceral abdominal analgesia in bariatric surgery: a report of 3 cases. Reg Anesth Pain Med. 2017;42(3):372-376. doi:10.1097/AAP.0000 000000000581

11. Melvin JP, Schrot RJ, Chu GM, Chin KJ. Low thoracic erector spinae plane block for perioperative analgesia in lumbosacral spine surgery: a case series. Can J Anaesth. 2018;65(9):1057-1065.

12. Celik M, Tulgar S, Ahiskalioglu A, Alper F. Is high volume lumbar erector spinae plane block an alternative to transforaminal epidural injection? Evaluation with MRI. Reg Anesth Pain Med. 2019;44 (9):906-907. doi:10.1136/rapm-2019-100514

13. Ivanusic J, Konishi Y, Barrington MJ. A cadaveric study investigating the mechanism of action of erector spinae blockade. Reg Anesth Pain Med. 2018;43(6):567-571. doi:10.1097/AAP.000000000000 0789

14. Tseng V, Xu JL. Erector spinae plane block for postoperative analgesia in lumbar spine surgery: is there a better option. J Neurosurg Anesthesiol. 2019. doi:10.1097/ANA.0000000000000631

15. Canturk M. Ultrasound-guided bilateral lumbar erector spinae plane block for postoperative analgesia after spondylolisthesis correction surgery. J Clin Anesth. 2019;57:77-78. doi:10.1016/j.jclinane.20 19.03.015

16. De Lara Gonzalez S, Basora MM, Tio M, Martinez-Camacho A, Fuster S, Sala-Blanch X. L4 erector spinal plane block after lumbar spine arthrodesi: a case-series. Rev Esp Anestesiol Reanim. 2019;66 (10):537-542.

17. Singh S, Chaudhary NK. Bilateral ultasound guided erector spinae plane block for postoperative pain management in lumbar spine surgery: a case series. J Neurosurg Anesthesiol. 2019;31(3):354. doi:10.1097/ANA.0000000000000518

18. Almeida CR, Oliveira AR, Cunha P. Continuous bilateral erector of spine plane block at T8 for extensive lumbar spine fusion surgery: case report. Pain Pract. 2019;19(5):536-540. doi:10.1111/papr.12774

19. Singh S, Choudhary NK, Lalin D, Verma VK. Bilateral ultrasound-guided erector spinae plane block for postoperative analgesia in lumbar spine surgery: a randomized control trial. J Neurosurg Anesthesiol. 2019.
20. Ueshima H, Inagaki M, Toyone T, Otake H. Efficacy of the erector spinae plane block for lumbar spinal surgery: a retrospective study. Asian Spine J. 2019;13(2):254-257. doi:10.31616/asj.2018.0114

21. Yayik AM, Cesur S, Ozturk F, et al. Postoperative analgesic efficacy of the ultrasound-guided erector spinae plane block in patients undergoing lumbar spinal decompression surgery: a randomized controlled study. World Neurosurg. 2019;126:e779-e785. doi:10.1016/j.wneu.20 19.02.149

22. Brandao J, Graca R, Sa M, Cardoso JM, Caramelo S, Correia C. Lumbar erector spinae plane block: successful control of acute pain after lumbar spine surgery - a clinical report. Rev Esp Anestesiol Reanim. 2019;66(3):167-171. doi:10.1016/j.redar.2018.10.005

23. Calandese F, Adduci A. Erector spinae plane block for acute postoperative pain management after anterior thoracolumbar spine surgery. J Clin Anesth. 2019;52:55-56. doi:10.1016/j.jclinane.2018.08.014

24. Cesur S, Yayik AM, Ozturk F, Ahiskalioglu A. Ultrasound-guided low thoracic erector spinae plane block for effective postoperative analgesia after lumbar surgery: report of five cases. Cureus. 2018;10(11):e3603.

25. Waldrop R, Cheng J, Devin C, McGirt M, Fehlings M, Berven S. The burden of spinal disorders in the elderly. Neurosurgery. 2015;77 (Suppl 4):S46-S50. doi:10.1227/NEU.0000000000000950

26. Graffigna G, Barello S. Patient Health Engagement (PHE) model in enhanced recovery after surgery (ERAS): monitoring patients' engagement and psychological resilience in minimally invasive thoracic surgery. $J$ Thorac Dis. 2018;10(Suppl 4):S517-S528. doi: $10.21037 /$ jtd.2017.12.84

27. De Cassai A, Bonvicini D, Correale C, Sandei L, Tulgar S, Tonetti T. Erector spinae plane block: a systematic qualitative review. Minerva Anestesiol. 2019;85(3):308-319. doi:10.23736/S0375-9393.18.13341-4

28. Perlas A, Chaparro LE, Chin KJ. Lumbar neuraxial ultrasound for spinal and epidural anesthesia: a systematic review and meta-analysis. Reg Anesth Pain Med. 2016;41(2):251-260. doi:10.1097/AAP.0000000000000184

29. Xu JL. Paraneuraxial nerve blocks: a well-defined novel terminology that is clinically essential for regional anesthesia. J Clin Anesth. 2017;43:14. doi:10.1016/j.jclinane.2017.09.002

30. Tseng V, Tara A, Hou J, Xu JL. Erector spinae plane block unbound: limits to safety in a patient with laminectomy. Saudi J Anaesth. 2019;13(3):253-254. doi:10.4103/sja.SJA_186_19

31. Gurkan Y, Aksu C, Kus A, Yorukoglu UH. Erector spinae plane block and thoracic paravertebral block for breast surgery compared to IV-morphine: a randomized controlled trial. J Clin Anesth. 2019;59:84-88. doi:10.1016/j.jclinane.2019.06.036

32. Tsui BC, Fonseca A, Munshey F, McFadyen G, Caruso TJ. The erector spinae plane (ESP) block: a pooled review of 242 cases. J Clin Anesth. 2019;53:29-34. doi:10.1016/j.jclinane.2018.09.036

33. Bianconi M, Ferraro L, Ricci R, et al. The pharmacokinetics and efficacy of ropivacaine continuous wound instillation after spine fusion surgery. Anesth Analg. 2004;98(1):166-172. doi:10.1213/01. ANE.0000093310.47375.44

34. Andreae MH, Andreae DA. Regional anaesthesia to prevent chronic pain after surgery: a cochrane systematic review and meta-analysis. Br J Anaesth. 2013;111(5):711-720. doi:10.1093/bja/aet213

35. Tulgar S, Yildirim A, Karaoglan A, Ozer Z. Erector spinae plane block as the main anesthetic method for peri-paravertebral area surgical procedure. J Clin Anesth. 2019;54:157. doi:10.1016/j. jclinane.2018.12.011

36. Fiorelli S, Leopizzi G, Saltelli G, et al. Bilateral ultrasound-guided erector spinae plane block for postoperative pain management in surgical repair of pectus excavatum via Ravitch technique. $J$ Clin Anesth. 2019;56:28-29. doi:10.1016/j.jclinane.2019.01.026

37. Breebaart MB, Van Aken D, De Fré O, et al. A prospective randomized double-blind trial of the efficacy of a bilateral lumbar erector spinae block on the $24 \mathrm{~h}$ morphine consumption after posterior lumbar inter-body fusion surgery. Trials. 2019;20(1):441. doi:10.1186/ s13063-019-3541-y 
38. Eisenstein TK. Opioids and the immune system: what is their mechanism of action. Br J Pharmacol. 2011;164(7):1826-1828. doi:10.1111/j.1476-5381.2011.01513.x
39. Cronin-Fenton DP, Heide-Jorgensen U, Ahern TP, et al. Opioids and breast cancer recurrence: a Danish population-based cohort study. Cancer. 2015;121(19):3507-3514. doi:10.1002/cncr.29532

\section{Publish your work in this journal}

The Journal of Pain Research is an international, peer reviewed, open access, online journal that welcomes laboratory and clinical findings in the fields of pain research and the prevention and management of pain. Original research, reviews, symposium reports, hypothesis formation and commentaries are all considered for publication. The manuscript

Submit your manuscript here: https://www.dovepress.com/journal-of-pain-research-journa management system is completely online and includes a very quick and fair peer-review system, which is all easy to use. Visit http:// www.dovepress.com/testimonials.php to read real quotes from published authors. 